\title{
ARGUMENTS
}

La rubrique "Arguments» offre un lieu de discussion et de confrontation.

«Arguments» souhaite contribuer à un dialogue scientifique fécond en publiant des réactions à diverses publications scientifiques. Ces pages sont également ouvertes aux réflexions suscitées par les dossiers de la revue.

\section{ÉLÉMENTS D'ORGANISATION GESTUELLE DES ICONES DE LOGICIELS ET D'ENVIRONNEMENTS INFORMATIQUES STANDARDISÉS}

Récemment D. Peraya ${ }^{1}$ a proposé une analyse sémiotique des icones de logiciels et d'environnements informatiques standardisés (ILEIS). Ce travail se fonde essentiellement sur une analyse de leur statut en tant qu'elles sont des représentations sémiotiques de nature analogique. Aussi l'objectif de cette première analyse a-t-il consisté à découvrir les éléments de leur syntaxe visuelle. Comme toute approche qui privilégie un aspect particulier, cette analyse ignore nécessairement d'autres aspects et parmi ceux-ci nous relèverons les différentes interactions avec les utilisateurs et la dynamique gestuelle qu'engendrent les ILEIS. C'est pourquoi, dans ce texte, nous désirons faire valoir une approche de cette dynamique gestuelle qui intègre certaines caractéristiques ergonomiques des icones. En conséquence, nous présenterons ici des éléments d'une articulation -d'une

\footnotetext{
${ }^{1}$ Voir D. Peraya, "Structures et fonctionnement sémiotiques des icones de logiciels et d'environnements informatiques standardisés (ILEIS)", Recherches en communication, $\mathrm{n}^{\circ} 10,1998, \mathrm{pp}$. 101-140.
} 
syntaxe ?- gestuelle complémentaire à celle, visuelle, élaborée initialement par cet auteur. Plus qu'une critique de ce texte, la présente contribution doit être lue comme le développement de certains aspects qui n'avaient pas été pris en compte initialement et sur lesquels, durant le processus de sa rédaction, nous avons trouvé un large accord ${ }^{1}$.

La classification dont nous suggérons ici les principes de base repose d'une part sur l'ergonomie et la fonction associée à chaque type d'ILEIS et d'autre part sur la richesse gestuelle potentielle de chacun de ces types. Nous insisterons sur la concordance que fait apparaître cette succincte analyse entre les caractéristiques ergonomiques et fonctionnelles des ILEIS et la complexité des modalités gestuelles qu'engendre leur manipulation par leur usager. On peut, de ce point de vue, établir quatre degrés fondamentaux que nous ordonnerons selon leur valeur décroissante. Dans un premier temps, nous développerons la classification fonctionnelle pour aborder, dans un second moment, les principes d'organisation et d'articulation gestuelles. Enfin, nous indiquerons quelques pistes sur la visualisation des états des ILEIS ainsi que sur les tendances actuelles.

\section{Une classification fonctionnelle des ILEIS}

Certaines de nos propres catégories correspondent à celles de nature fonctionnelle proposées par Peraya dans son article : les deux types d'icones de fonction (icones outils et icones de commandes) d'une part et les icones de marquage d'autre part. Par contre, notre point de vue nous impose de créer d'autres classes ou encore de redistribuer autrement celles proposées. Prenons un exemple : du point de vue de l'analyse des représentations visuelles, une icone analogique comme celle d'un dossier, ou d'une disquette que l'on connait par l'environnement du bureau peuvent se voir traiter de la même façon que les boutons affichant les représentations identiques : elles sont des représentations de même nature et une syntaxe identique préside à leur construction sémio-pragmatique. Pourtant des points de vue ergonomique et gestuel, les comportements qu'elles rendent possibles pour

1 Ce texte est le résultat d'une discussion menée au sein de l'Unité TECFA à propos de ce texte et de la conception des fonctions, du statut sẹmiotique, enfin du modus significandi des ILEIS. La version finale du texte commun que nous publions constitue une étape et une contribution à ce débat. 
l'utilisateur sont, nous le verrons, très divers. Enfin, leurs fonctions aussi diffèrent. Aussi, de notre point de vue, on ne peut ni les considérer de façon identique ni les catégoriser sous un label unique.

Enfin, il est important de remarquer que le terme même d'icone est profondément ambigu puisque du point de vue sémio-pragmatique il représente un type de représentation tandis que des points de vue ergonomique, fonctionnel et gestuel, il désigne une des modalités d'interaction homme/machine parmi d'autres (boutons, menus déroulants, menus contextuels, etc.) ${ }^{1}$.

\section{Les icones d'élément}

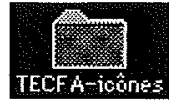

Ces ILEIS se présentent comme la réification, la manifestation manipulable et visible à l'écran d'un objet logique, d'un élément : par exemple, un fichier, un dossier, une application, un disque, un périphérique, etc. Définies pour la toute première fois par Apple pour les développeurs sur Macintosh ${ }^{2}$, elles possèdent le plus grand potentiel gestuel et présentent une articulation et une organisation gestuelles les plus développées : l'utilisateur peut les glisser vers d'autres, définissant ainsi un objet, une action et ses compléments, il peut les sélectionner en vue d'une action, les "double-cliquer" pour ouvrir l'objet auquel renvoie l'icone, etc. Nous les décrirons plus loin.

Cette première catégorie était absente de la classification présentée par Peraya parce que, privilégiant l'analyse de la structure sémiotique des ILEIS et donc des formes de représentation visuelle, elle ne pouvait évidemment pas tenir compte ni de l'interaction ni de la manipulation des différents objets visibles à l'écran qui font appel à l'ergonomie et à la gestuelle.

1 On sait que ce type d'interface a été développé au Palo Alto Research Center (PARC) de XEROX au début des années 80.

2 APple, Apple Interface guidelines. The Apple desktop interface, Cupertino, Ed. Apple Computer, 1986. 


\section{Les ILEIS de fonction}

\subsection{Les boutons}

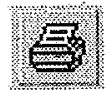

\section{Enregistrer}

Nous trouvons sous cette catégorie, les icones d'actions qui représentent un verbe d'action tel que imprimer, couper, coller, etc. Ces ILEIS sont, pour l'utilisateur, des supports visibles d'actionnement ou d'activation de fonction du système'. Elles correspondent aux icones de commandes définies par Peraya que l'on trouve normalement dans les palettes d'icones, dans les barres de commandes et de navigation, etc. Elles peuvent prendre deux formes : les boutonstexte et les boutons-icones. La gestuelle qu'autorisent ces boutons est, nous le verrons, de loin moins complexe et constitue le second degré de l'échelle proposée.

\subsection{Outils}

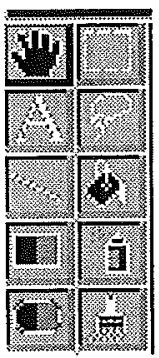

A cette catégorie appartiennent les icones qui permettent de choisir une modalité de fonctionnement. Ce sont les icones des palettes d'outils qui sont apparues pour la première fois dans l'ancêtre MacPaint. Les ILEIS-outils modifient le système et activent un mode de fonctionnement particulier : le curseur perd son rôle de "prothèse déictique et/ou préhensile" pour se muer en un outil dont l'effet conserve la trace du geste et l'inscription d'une trace manuelle à travers la tâche réalisée. Cette catégorie correspond à celle des icones outils identifiée dans l'article déjà cité ${ }^{2}$.

1 Les boutons -du moins ceux des palettes de commande- peuvent être comparés aux articles de menus par leur fonctionnalité, mais en plus visible. Souvent (dans les logiciels tels que Word, ClarisWorks, etc.) les boutons des palettes reprennent les articles de menus les plus fréquents. Les deux définissent une action dont les paramètres sont implicites. On peut rappeler enfin que les menus offrent la possibilité de distinguer les actions directes ("Imprimer", "Copier", etc.) des appels de dialogues ("Ouvrir..." noter les points de suspension) qui font apparaître une fenêtre de dialogue pour parcourir l'arborescence du disque et sélectionner un fichier à ouvrir.

${ }^{2} \mathrm{La}$ différence entre commande et outil mériterait de plus longs développements. Rappelons qu'elle reprend pour l'instant la distinction opérée par de nombreux auteurs entre la peinture et la photographie ou encore à la chirographie. Voir à ce sujet R. BARTHEs, "Rhétorique de l'image", Communications, n 4, 1964. 
Remarquons que selon l'outil sélectionné, les mêmes actions produiront des effets différents. On parle dans ce cas d'une interface modale. Par exemple, si l'on a choisi l'outil pinceau, le geste sur la surface de travail produit une trânée de pixels de la couleur et du motif choisis, dont l'épaisseur est paramétrable. Si le crayon est actif, on aura une ligne de pixels. Le même geste a des effets différents selon le mode de travail choisi.

Bien que fonctionnellement et ergonomiquement distinctes des boutons, les ILEIS-outils obéissent à une logique gestuelle fort semblable.

\section{Les icones de marquage}

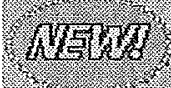

Ces ILEIS précisent le sens, indiquent le statut d'un texte ou d'un autre élément graphique. Autrement dit, elles "font partie des outils disponibles pour la mise en texte et l'ergonomie textuelle"1. Autrement dit elles possèdent une fonction métatextuelle et ne participent d'aucune interaction homme/machine. Elles ne possèdent donc aucune fonctionnalité au sens où nous l'entendons ici et, logiquement, constituent le degré zéro de la gestualité.

\section{Éléments d'articulation gestuelle}

\section{Les icones d'élément}

Les icones d'élément permettent une articulation syntaxique assez évoluée, qui évoque d'une certaine manière la structure générale du langage articulé : un sujet, un verbe, un objet et des éléments définissant le contexte et les circonstances.

- Le sujet est constitué par l'utilisateur qui manipule à travers la souris les différents objets visibles à l'écran. La manipulation des

W. BENJAMIN, “L'œuvre d'art à l'époque de sa reproductibilité technique”, Sur l'art et la photographie, Paris, Art\&esthétique, 1997, p. 20 (publication originale en 1936). C. CossETTE, Les image démaquillées : approche scientifique de la communication par l'image, Québec, Éditions Riguil, 1983, pp. 65 et sv. Cités par

D. PERAYA, op. cit.

1 D. PERAYA, op. cit., p. 112. 
icones relève donc toujours d'un processus d'énonciation au sens strict puisqu'il inclut le sujet.

- L'objet sur lequel porte l'action est défini par l'icone que le curseur saisit (en enfonçant le bouton de la souris après avoir positionné le curseur sur l'icone de l'objet). Le positionnement fait partie intégrante de la gestuelle.

- Le verbe d'action est défini par le geste et donc par le déplacement de la souris auquel correspond un glissement du curseur. Pourtant le verbe d'action ne prend réellement son sens que contextuellement à partir de la trajectoire décrite par le geste et de sa cible, une autre icone ou une fenêtre. Notons que dans ce processus de sémantisation, c'est la cible qui paraît jouer le rôle essentiel. Elle transforme en effet un déplacement aléatoire en un mouvement orienté et donc intentionnel : elle constitue dès lors le mouvement en une trajectoire et, ce faisant, lui donne son sens.

Par exemple glisser l'icone d'un fichier vers une partition de disque, un répertoire, une disquette, etc. définit le geste comme l'action de copier ce fichier dans cette partition, ce répertoire, cette disquette, etc. Déplacer l'icone d'un fichier exécutable, un fichier Word par exemple, vers la fenêtre ouverte de ce même logiciel, signifiera "ouvrir le fichier désigné par l'icone saisie et déplacée". On le voit, le geste ne peut guère être dissocié de sa cible, qui contextualise l'action.

- Les éléments de contextualisation, qui sont attachés à l'icone choisie comme cible. Par exemple glisser l'icone d'un fichier de traitement de texte vers une icone d'imprimante décrit l'action d'imprimer le fichier sélectionné et déplacé par une imprimante particulière dont, par exemple les différents réglages (couleur ; qualité d'impression soit standard, économique ou de haute qualité ; choix du tiroir à papier, \% de la taille de la feuille, etc.) ont été définis lors de la création de cette icone particulière. On peut avoir plusieurs icones d'imprimante correspondant à des imprimantes virtuelles, chacune d'elles présentant des réglages d'impression différents. Le choix de celle sur laquelle l'utilisateur glisse le fichier à imprimer déterminera alors les réglages qu'il aura retenus.

Les icones d'élément peuvent faire l'objet d'un second type de gestuelle : le "double clic" dont le degré de complexité est moindre. Le sujet demeure l'utilisateur, l'objet est constitué de l'élément 
sélectionné et le verbe par défaut est l'action d'ouverture. Le doubleclic est une réminiscence des premiers temps des interfaces graphiques : aux débuts de la Lisa-le prédécesseur de Macintosh-, on sélectionnait un élément, puis on choisissait le menu correspondant ("Imprimer", "Ouvrir", etc.). Mais l'article "Ouvrir" était tellement fréquemment employé qu'un raccourci a été développé : le double-clic prévu à l'origine pour les usagers avancés.

Quant aux alias et en partie aux raccourcis, ils sont une seconde représentation d'un objet et possèdent donc toutes les propriétés de celui-ci. En principe, ils sont visualisés par la même icone que l'objet même si du point de vue logique du système ils ne font que renvoyer à l'objet original.

\section{Les boutons et les outils}

\subsection{Une gestuelle réduite}

L'articulation gestuelle est ici réduite à sa plus simple expression. La gestuelle -le positionnement sur un bouton (désignation) et un clic (sélection)-, définit uniquement le choix de l'objet : l'objet est ce qui est désigné et sélectionné, ou dans certains cas, ce qui se trouve au premier plan, un document, un texte par exemple. Un clic sur le bouton déclenche une action, active une fonctionnalité particulière. Nous pourrions avancer cette formule lapidaire qui résume fort bien la part du gestuel dans l'interaction : "positionner et cliquer = activer une fonction".

Pourtant contrairement à ce qui se passait dans le cas des icones d'élément, le gestuel ne peut en rien définir ou préciser le verbe. Dans son analyse, Peraya a montré que, dans le cas de ces icones de commande, le verbe -l'action ou la fonction- était défini le plus souvent par un processus de sémantisation par contiguilté de type métonymique. C'est la représentation analogique de l'instrument, de l'outil qui fait l'action qui détermine le sens de cette dernière : la représentation de l'imprimante sur le bouton conduit à signifier l'action d'imprimer; celle de la disquette, amène par une double métonymie, à signifier l'action de sauver. Enfin, les éventuels éléments de contextualisation sont implicites : imprimer sur l'imprimante par défaut, avec les réglages par défaut, par exemple. De la même façon que pour la définition du verbe, la gestuelle ne joue ici aucun rôle. 
Du point de vue de la gestuelle, les outils se comportent de façon identique $^{1}$. L'articulation gestuelle apparaît tout autant réduite au minimum. Un clic active ou désactive le mode de travail correspondant : pinceau ou crayon...

Pour rendre compte de la gestuelle caractéristique des outils, nous adapterons simplement la formule en ces termes : "positionner et cliquer $=$ changer de prothèse et adopter une prothèse outil". Si l'on compare ces deux formules, on comprendra aisément que, envisagés du point de vue de la gestuelle, boutons et outils sont comparables. Pourtant des points de vue ergonomique et fonctionnel, dans le premier cas il s'agit, pour l'utilisateur, de faire faire au système une opération définie par défaut (valeur factitive) tandis que dans le second, il s'agit pour lui de modifier son interface de travail et la fonction d'un périphérique - la souris - afin de faire lui-même. La différence devient alors primordiale.

\section{2. États visuels des icones}

Au plan ergonomique, on peut noter que les boutons et les outils se distinguent encore par le mode d'affichage de leur état, autrement dit par le type des rétroactions visuelles qu'ils renvoient à l'usager à la suite de son action et de son comportement gestuel. Les états visuels des ILEIS ne concernent pas, stricto sensu, l'articulation gestuelle qui leur est liée. Pourtant, dans la mesure où ils en constituent la rétroaction immédiate et dès lors un indicateur permettant de réguler l'interaction, nous pensons légitime d'en analyser quelques éléments sous cette rubrique. Par exemple un bouton paraît s'enfoncer quand le bouton de la souris est appuyé alors que le curseur est au-dessus de l'icone-bouton. L'action n'est pas déclenchée à ce stade, et si le curseur est glissé en dehors du bouton, rien ne sera effectué. L'action ne sera déclenchée que si le bouton est relâché alors que le curseur est encore "dans" l'icone-bouton.

Boutons et outils possèdent donc tous au minimum deux états : neutre et enfoncé comme le montrent les deux icones ci-dessous. Le

1 En toute rigueur terminologique, on devrait conserver l'opposition actif/inactif pour les boutons et l'opposition sélectionné/non sélectionné pour les outils. On spécifierait de cette façon les caractéristiques des boutons et des outils: respectivement la réceptivité et l'état. Ces deux oppositions pourraient être considérées comme des variantes contextuelles de l'opposition fondamentale : disponible/non disponible. 
plus souvent, depuis la création de l'interface $N E X T S T E P^{1}$ et de son interface relief sur fond gris, un effet d'ombrage et de perspective donne l'illusion d'un état enfoncé ou non.

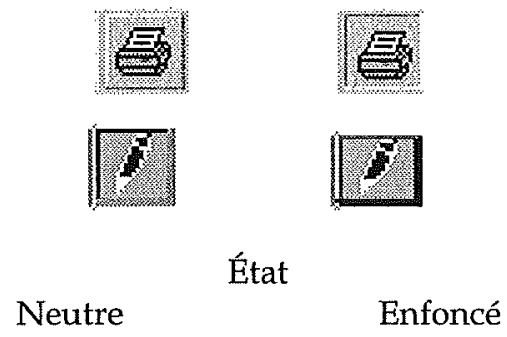

Pourtant, les boutons connaissent parfois des états supplémentaires : ils apparaissent grisés quand la fonction correspondante est indisponible et qu'ils sont en conséquence inactifs. Dans ce cas, ils apparaissent comme un indicateur de l'état global du système. Parfois même, ils connaissent deux états supplémentaires : inactif neutre et inactif enfoncé. Enfin, dans les interfaces récents, un bouton apparaît en relief lorsque le curseur le survole. Cette dernière tendance s'est même généralisée aux outils comme c'est le cas par exemple dans la dernière version du logiciel graphique PSP5.

Cette dernière façon de faire, qui a pour but de manifester à l'utilisateur la présence d'un objet cliquable, renforce un facteur de rétroaction et de régulation de la gestuelle : la modification de la forme du curseur dès qu'il passe "au-dessus" de la zone sensible du bouton, avant même que l'on ne le clique. Par exemple, le curseur de la souris se mue en une "main, l'index tendu"2.

Notons que dans les premières interfaces graphiques, le bouton avait une forme codifiée très précisément, mais avec l'avènement du multimédia la diversification des formes que pouvait manifester son support visuel -les icones lui servant de support explicite- a nécessité une autre manière de manifester sa présence ; l'usager s'est donc vu contraint de chercher par une gestuelle exploratoire -"à tâtons"- les boutons et les découvrait par cette rétro-information. Dans de nombreux jeux informatiques d'aventure, dans lesquels la découverte

1 Il s'agit d'un Operating System très avancé développé par Steve Jobs à la fin des années 80 .

2 Cette modalité est née sans doute avec Hypercard et son usage est largement répandu avec l'extraordinaire développement des hypertextes dans les CD-ROM et le WEB. 
constitue l'une des activités et des motivations essentielles, le procédé est exploité systématiquement. En conclusion, on peut affirmer que l'état visuel des boutons et des outils ainsi que les modifications du curseur constituent des indicateurs forts pour l'interaction et la régulation de la gestuelle.

Deux remarques encore. Il faut distinguer cependant les différents état visuels des ILEIS selon qu'ils servent de guide à la gestuelle ou qu'ils manifestent l'état global du système. Aujourd'hui, le bouton de l'impression demeure enfoncé après le clic de déclenchement de l'action et ce durant le temps de la préparation du fichier. Un outil demeure "enfoncé" tant que l'outil est utilisé. Dans ces cas, c'est bien une information sur l'état du système qui est fournie à l'utilisateur et la gestuelle n'est plus guère concernée. On peut faire la même remarque à propos des différentes formes que peut prendre le curseur : tantôt elles guident le comportement gestuel de l'utilisateur et tantôt elle lui fournissent de l'information sur l'état global du système : tant que se prépare l'impression d'un fichier, le curseur se mue en imprimante. Autrement dit, aussi longtemps que dure l'action enclenchée par le clic, la forme du curseur témoigne du déroulement de cette action ainsi que de sa nature (une imprimante pour "imprimer", un fichier qui entre dans un dossier pour "sauver", etc.). Dans le cas des outils, on observe une transformation identique : le curseur prend une forme évoquant l'outil choisi et donne donc une confirmation du mode de travail actif. Ceci est particulièrement important puisque, dans une interface modale, l'usager doit avoir sous les yeux un rappel constant de la modalité active afin de savoir quels effets auront les actions qu'il va entreprendre. De ce point de vue, le fonctionnement du bouton et de l'outil se distingue par le type de rétro-information donnée à l'usager : respectivement, l'activité du système sur le mode du tout ou rien ou la modalité de fonctionnement choisie.
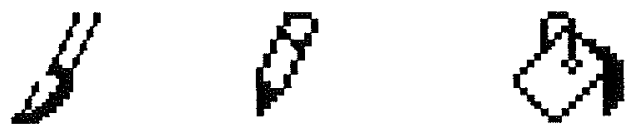

Exemples de curseurs outils

\section{Les icones de marquage}

Elles ne permettent par définition aucun traitement gestuel. 


\section{Conclusion et prospective}

Nous pensons avoir montré la diversité des modalités d'articulation gestuelle en fonction des différents types fonctionnels et ergonomiques d'ILEIS. Les icones d'éléments sont celles qui présentent le plus grand potentiel tandis que les icones de marquage en sont totalement dépourvues. Au niveau intermédiaire, boutons et outils présentent une gestuelle limitée, souvent guidée par des modalités visuelles : affichage de l'état du bouton ou de l'outil et modification de la forme du curseur. Enfin, nous pensons indispensable de distinguer deux cas parmi les états visuels et les modifications de curseur : ceux qui guident la gestuelle et ceux qui informent l'utilisateur de l'état du système qu'il s'agisse d'une action en cours ou du choix d'une modalité de travail.

Parmi les tendances et l'évolution que révèle l'observation des logiciels et des environnements informatiques standardisés, rappelons tout d'abord l'assimilation des boutons aux outils et leur traitement identique du point de vue de l'affichage de leurs différents états. Ensuite, il semble que la généralisation de l'utilisation des boutons et du curseur à travers la diversité de ses formes comme indicateurs de l'état du système soit assez récente.

Enfin on note, sans doute est-ce lié à l'extraordinaire développement du WEB, que les modalités d'interaction passent de plus en plus par des boutons ou par les boutons implicites que sont les liens soulignés du WEB et les zones sensibles. La richesse du langage articulé émergeant que permet la gestuelle caractéristique des icones d'élément pourrait bien céder le pas à une interaction assez primaire, celle du clic. Autrement dit, la fonction déictique/préhensile du curseur de la souris suffirait à gérer l'ensemble des interactions. En termes sémiotiques, on observerait donc un mouvement de régression des formes symboliques vers celles primaires liées aux signes indiciels ${ }^{1}$.

Est-ce un signe de l'accès par un public de plus en plus large à l'informatique, ou le reflet d'une infantilisation de l'usager par les grands constructeurs?

François LOMBARD et Daniel PERAYA ${ }^{2}$

1 Voir à ce propos D. Bougnoux, La communication par la bande, Paris, La Découverte, 1991.

2 TECFA, Unité des technologies éducatives, Faculté de psychologie et des sciences de l'éducation, Université de Genève. 